\title{
A proof of the Kauffman-Harary Conjecture
}

\author{
THOMAS W MATTMAN \\ PABLO SOLIS
}

\begin{abstract}
We prove the Kauffman-Harary Conjecture, posed in 1999: given a reduced, alternating diagram $\mathcal{D}$ of a knot with prime determinant $p$, every nontrivial Fox $p$-coloring of $\mathcal{D}$ will assign different colors to different arcs.
\end{abstract}

$57 \mathrm{M} 25$

\section{Introduction}

In 1999, Louis Kauffman and Frank Harary published a paper [5] detailing a graphtheoretic approach to the study of knot theory. In the paper they state a conjecture (Alternation Conjecture 6.2) that has come to be known as the Kauffman-Harary Conjecture:

Conjecture 1 (Kauffman-Harary Conjecture) Let $\mathcal{D}$ be a reduced, alternating diagram of the knot $k$ having prime determinant $p$. Then, every nontrivial $p$-coloring of $\mathcal{D}$ assigns different colors to different arcs.

This provides a nice connection between two knot invariants, the determinant, which is relatively easy to calculate, for example, using the Alexander polynomial, and the least number of colors required on a minimal diagram of a knot, an invariant which is, in general, very difficult to evaluate. The conjecture asserts that in the case of an alternating knot, if the determinant is prime, then the least number of colors over minimal diagrams is simply the crossing number. Conjecture 1 is known to hold for rational knots by Kauffman and Lambropoulou [7] and Person et al [12], pretzel knots by Asaeda, Przytycki and Sikora [2], and many Turk's head knots by Dowdall et al [4]. Our goal in this paper is to prove the conjecture for all alternating knots of prime determinant.

In order to give an overview of our approach we recall some basic ideas about coloring. Let $k$ be an alternating knot of prime determinant $p$. A (Fox) $p$-coloring of a diagram $\mathcal{D}$ of $k$ is an assignment of integers $\bmod p$ to the arcs of $\mathcal{D}$ such that the equation $2 x-y-z \equiv 0$ mod $p$ holds at each crossing, $x$ being the color of the over 
arc, while $y$ and $z$ are the colors of the two under arcs. It's convenient to encode this requirement as an $n \times n$ matrix $C^{\prime}$, the crossing matrix, where $n$ is the number of crossings in $\mathcal{D}$. Each row of $C^{\prime}$ corresponds to a crossing and has one 2 entry and two -1 's, the other entries being 0 . The columns of $C^{\prime}$ then correspond to $\operatorname{arcs}$ of $\mathcal{D}$ and colorings to vectors $X$ such that $C^{\prime} X \equiv \overrightarrow{0}$ modulo $p$.

Now, every constant vector $X=(c, c, c, \ldots, c)$ gives a coloring, but if we move to the minor $C$ defined by removing the last row and column of $C^{\prime}$, we will have a way of identifying nontrivial colorings. It turns out that $|\operatorname{det} C|=\operatorname{det} k$, the knot's determinant, which is a knot invariant. As $\operatorname{det} k=p$, a prime, $C$ is invertible over the rationals. We define the coloring matrix $L=p C^{-1}$ as the classical adjoint of $C$. In Section 3, we argue that each column of $L$ includes entries that are not zero modulo $p$. The argument depends on the observation that, as $k$ is alternating and has prime determinant, it is a prime knot.

As no column of $L$ is zero modulo $p$, there will be heterogeneous colorings of $\mathcal{D}$, where a coloring is heterogeneous if it assigns different colors to different arcs. To complete the argument, we use the fact that the nullity of $C$ is one to conclude that if one nontrivial coloring is heterogeneous, then they all are.

The structure of our paper is as follows. In Section 2, we briefly discuss colorability and crossing matrices and present preliminary definitions and notation used throughout the paper. We also prove two lemmas, one showing that appending a zero to a vector in $C$ 's null space gives a vector in $C^{\prime}$ 's null space, while the other demonstrates that taking the mirror reflection of an alternating diagram corresponds to transposing the crossing matrix. In Section 3, we introduce the coloring matrix $L$ and develop the properties of colorings constructed from its columns. This culminates in a proof that every column of $L$ includes entries that are not zero modulo the determinant. Finally, in Section 4, we prove the conjecture.

\section{Preliminaries}

In this section, we review basic results on colorability; references for this material include Livingston [8], Mo [9], and Przytycki [13]. We end the section with two lemmas.

Following [8], a diagram of a knot is a planar representation with gaps left to show where the knot crosses under itself; for example, see Figure 1 below. Let $[k]$ denote the set of diagrams of a knot $k$. We say that a diagram is reduced if it has no nugatory crossings (see Adams [1, Chapter 3], for example). Let $\left[k_{r}\right]$ denote the subset of $[k]$ 
consisting of all reduced diagrams. We say $k$ is $n$-crossing if some $\mathcal{D} \in[k]$ has $n$ crossings and no other $\mathcal{D}^{\prime} \in[k]$ has fewer than $n$ crossings. It's known (by Kauffman [6], Murasugi [10] and Thistlethwaite [14]) that if $k$ is an $n$-crossing alternating knot, then every $\mathcal{D}$ in $\left[k_{r}\right]$ is alternating and has $n$ crossings and $n$ arcs.

We will now define $p$-coloring where $p$ is an integer greater than $1, k$ is a knot, and $\mathcal{D} \in[k]$. Let $x, y, z$ denote integers which label the over arc and two under arcs, respectively, at a crossing of $\mathcal{D}$. The crossing satisfies the condition of $p$-colorability if

$$
2 x-y-z \equiv 0 \bmod p .
$$

We say $k$ is $p$-colorable if there is some $\mathcal{D} \in[k]$ such that the arcs of $\mathcal{D}$ can be labeled, or colored, with the numbers $0, \ldots, p-1$ so that at least two numbers are used and every crossing satisfies the condition of $p$-colorability. The numbers $0, \ldots, p-1$ are called colors. The colors assigned to the arcs such that at each crossing the condition of $p$-colorability is satisfied, make up a $p$-coloring of $\mathcal{D}$. A $p$-coloring where every arc is assigned the same color is called a trivial coloring.

Let $\mathcal{D} \in\left[k_{r}\right]$ be an $n$-crossing diagram of the knot $k$. A labeling of $\mathcal{D}$ is a particular indexing of the crossings $\left\{c_{1}, \ldots, c_{n}\right\}$ and of the $\operatorname{arcs}\left\{a_{1}, \ldots, a_{n}\right\}$. Given $\mathcal{D}$ and some labeling, we define the crossing matrix $C^{\prime}$ as

$$
C_{i j}^{\prime}= \begin{cases}2 & \text { if } a_{j} \text { is the over arc at crossing } c_{i}, \\ -1 & \text { if } a_{j} \text { is an under } \operatorname{arc} \text { at } \operatorname{crossing} c_{i}, \\ 0 & \text { otherwise }\end{cases}
$$

Owing to (1) and (2), it follows that a $p$-coloring can be represented by a vector $X^{\prime}$ such that $C^{\prime} X^{\prime} \equiv \overrightarrow{0} \bmod p$.

The matrix $C^{\prime}$ provides a straightforward way to compute the determinant of a knot. In particular, let $C$ be any $(n-1) \times(n-1)$ minor of $C^{\prime}$. We say $C$ is a minor crossing matrix. The determinant of the knot $k$ is the absolute value of the determinant of $C$ : $\operatorname{det} k=|\operatorname{det} C|$. It follows that $k$ is $p$-colorable if and only if $\operatorname{gcd}(p, \operatorname{det} k)>1$. Given $C^{\prime}$, there are $n^{2}$ choices for $C$, but, to avoid ambiguity, we will reserve the symbol $C$ for the minor crossing matrix obtained by removing the last column and last row of $C^{\prime}$. In this paper we will be investigating det $k$-colorings of diagrams of $k$ and, for simplicity, we will usually just say "coloring" when the value det $k$ is clear from the context.

We conclude this section with two lemmas. The first shows that, by appending a zero, a vector in the null space of $C$ can be "extended" to a coloring. 
Lemma 2.1 Let $k$ be a knot with reduced diagram $\mathcal{D} \in\left[k_{r}\right]$ and minor crossing matrix $C$. A vector $X$ such that $C X \equiv \overrightarrow{0} \bmod \operatorname{det} k$, can be extended to a coloring of the diagram $\mathcal{D}$ by adding a zero as the last entry. That is,

$$
C^{\prime}\left(\begin{array}{c}
X \\
0
\end{array}\right) \equiv \overrightarrow{0} \bmod \operatorname{det} k
$$

Proof It is clear why the first $n-1$ entries of the product in (3) should be 0 mod det $k$. The last entry is also 0 because the last row of $C^{\prime}$ can be expressed in terms of the first $n-1$ rows. Indeed, if $k$ is an alternating knot and $r_{i}$ are the rows of its crossing matrix then $r_{j}=-\sum_{i \neq j} r_{i}$. More generally, Livingston [8] shows that, for any knot, each row of the crossing matrix can be expressed as a linear combination of the other rows. Thus, the last entry of (3) is also zero.

Our second lemma shows a connection between the crossing matrices of two diagrams related by a mirror reflection. Fix a specific diagram $\mathcal{D} \in\left[k_{r}\right]$ of an alternating knot $k$ and use the following procedure to label its arcs and crossings: After orienting the knot, pick an arc, and label it $a_{1}$. Following the orientation, label the next $\operatorname{arc} a_{2}$. The crossing that has $a_{1}$ and $a_{2}$ as under arcs is labeled $c_{1}$. Continuing in this way, the $i$-th arc we reach is labeled $a_{i}$ and the crossing that has $a_{i-1}$ and $a_{i}$ as under arcs is labeled $c_{i-1}$. Finally, the crossing between $\operatorname{arcs} a_{n}$ and $a_{1}$ is labeled $c_{n}$, where $n$ is the number of crossings in $\mathcal{D}$. We call such a labeling an oriented labeling.

For an oriented labeling of an alternating diagram with $n$ arcs, the expression $2 a_{j}-$ $a_{i}-a_{i+1}$, where $i<n$, which can be read off the $i$-th row of the crossing matrix, expresses that $a_{j}, a_{i}, a_{i+1}$ are the arcs present in crossing $c_{i}$. For $i=n$, we have $2 a_{j}-a_{1}-a_{n}$. Similarly, $2 c_{l}-c_{j}-c_{j-1}$, for $j>1$, can be read off the $j$-th column of the crossing matrix, and expresses that the arc $a_{j}$ is an over arc at the crossing $c_{l}$, and is an under arc at the crossings $c_{j}, c_{j-1}$. For $j=1$, the expression is $2 c_{l}-c_{1}-c_{n}$.

Given $\mathcal{D}$, its mirror image $\mathcal{D}^{m}$ is the diagram obtained by reversing all the crossings; that is, we change the over crossings to under crossings and vice versa. Oriented labelings of $\mathcal{D}$ correspond to oriented labelings of $\mathcal{D}^{m}$ : for a crossing of $\mathcal{D}$ with over $\operatorname{arc} a_{j}$ and under $\operatorname{arcs} a_{i}, a_{i+1}$, the corresponding crossing in $\mathcal{D}^{m}$ has $a_{i}$ as the over arc, and $a_{j-1}, a_{j}$ as the under arcs. (Here we are taking indices modulo $n$, the crossing number. We will frequently do this in what follows.)

In summary, the arc $a_{i}$ of $\mathcal{D}^{m}$ is an over arc at crossing $c_{j-1}$, and, as always, $a_{i}$ is an under arc at crossings $c_{i-1}$ and $c_{i}$. Thus, the crossing $c_{i}$ in $\mathcal{D}$ given by $2 a_{j}-a_{i}-a_{i+1}$ transforms to the arc $a_{i}$ in $\mathcal{D}^{m}$ given by $2 c_{j-1}-c_{i-1}-c_{i}$. More concisely, $a_{j}$ and $c_{i}$ in $\mathcal{D}$ correspond to $c_{j-1}$ and $a_{i}$ in $\mathcal{D}^{m}$, respectively. This leads to the following lemma. 
Lemma 2.2 Let $k$ be an alternating knot. Let $C^{\prime}$ be a crossing matrix for $\mathcal{D} \in\left[k_{r}\right]$. Then the matrix $C^{\prime \top}$ is a crossing matrix for $\mathcal{D}^{m}$.

Proof Give $\mathcal{D}$ an oriented labeling. Permute the rows of $C^{\prime \top}$ by sending the $i$-th row to the $(i-1)$-st row (for each $i>2$ ) and the first row to the $n$-th row; call this matrix $D$. As in the discussion above, $D$ is the crossing matrix for an oriented labeling of $\mathcal{D}^{m}$. However, the set of crossing matrices of a diagram is closed under row and column permutations, so $C^{\prime \top}$ is also a crossing matrix for $\mathcal{D}^{m}$.

\section{The main result on the coloring matrix}

Let $k$ be an alternating knot of prime determinant and $\mathcal{D} \in\left[k_{r}\right]$. Whichever $\mathcal{D}$ we choose, $|\operatorname{det} C|=\operatorname{det} k$, a knot invariant, so that $C$ has prime determinant and is therefore invertible over the rationals. We define $L=\operatorname{det} k \cdot C^{-1}$ and call $L$ the coloring matrix. In this section we will prove that every column of $L$ contains entries that are not zero modulo det $k$.

Our strategy is to argue by contradiction. We will show that if there is a column of zeroes, then there must be a "pseudo coloring" $Y^{\prime}$. This is a way of labeling the arcs of $\mathcal{D}$ such that the coloring condition (1) fails at exactly two crossings, one, call it the $+1-$ crossing, where $2 x-y-z=1$ and another, the -1 -crossing, where $2 x-y-z=-1$. We then investigate the properties of $Y^{\prime}$ eventually deducing that $Y^{\prime}$ exists only if $\mathcal{D}$ is the diagram of a connected sum of two knots. (The distinguished $+1-$ and -1 -crossings appear in distinct components.) This is a contradiction; since $k$ is alternating and has prime determinant, it is in fact prime.

We begin with some of the properties of the coloring matrix. The entries of $L$ are signed minors of $C$ and, therefore, integers. Note that $C L$ and $L C$ both give the zero matrix mod det $k$. In particular, if $w_{i}$ is the $i-$ th column of $L$, then $C w_{i} \equiv \overrightarrow{0}$ $\bmod \operatorname{det} k$. We will use contradiction to show that $w_{i} \neq \equiv \overrightarrow{0} \bmod \operatorname{det} k$.

Lemma 3.1 Let $k$ be an $n$-crossing alternating knot and let $\mathcal{D} \in\left[k_{r}\right]$. If the $j$-th column of $L$ is $\overrightarrow{0} \bmod \operatorname{det} k$, then there is a pseudo coloring $Y^{\prime}$ of $k$ such that $Y^{\prime}$ has all positive entries and $C^{\prime} Y^{\prime}=e_{j}-e_{n}$.

Proof Let $L=\left(w_{1} \cdots w_{n-1}\right)$ be the coloring matrix of an alternating knot $k$. For some $j<n$ we have $w_{j} \equiv \overrightarrow{0} \bmod \operatorname{det} k$. Equivalently, the $j$-th column of $C^{-1}$ has all integer entries. Let $Y$ be the $j$-th column of $C^{-1}$, ie, $Y=(1 / \operatorname{det} k) w_{j}$. Then, from the equation $C C^{-1}=I$, we infer that $C Y=e_{j}$. Set

$$
Y^{\prime}=\left(\begin{array}{l}
Y \\
0
\end{array}\right) \text {. }
$$


As in the proof of Lemma 2.1, we find that

$$
C^{\prime} Y^{\prime}=e_{j}-e_{n},
$$

where we now consider $e_{j}$ to be a vector of $\mathbb{R}^{n}$. Let $T$ denote the vector of all 1 's, that is, a trivial coloring. To ensure that $Y^{\prime}$ has all positive entries we note that $C^{\prime} T=\overrightarrow{0}$, hence $C^{\prime}\left(Y^{\prime}+m T\right)=e_{j}-e_{n}$. So, choose $m$ large enough to ensure that $Y^{\prime}+m T$ has all positive entries, and take that to be $Y^{\prime}$.

Remark The pseudo coloring $Y^{\prime}$ described by the lemma is not a trivial coloring. A trivial coloring would give $C^{\prime} Y^{\prime}=\overrightarrow{0}$.
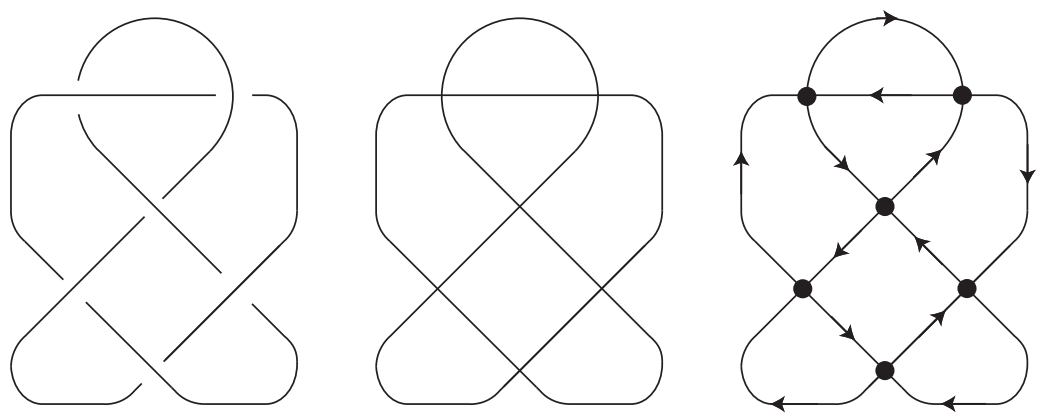

Figure 1: From left to right: a diagram $\mathcal{D}$ of an alternating knot, "filling in" the gaps, the graph $G_{\mathcal{D}}$

By "filling in" the gaps of the diagram $\mathcal{D}$ of an alternating knot, we have a regular projection of the knot $k$. We will view this as a four valent planar graph $G_{\mathcal{D}}$ by placing vertices at each crossing. As in Figure 1, we make a directed graph by orienting the "under arc" edges away from the crossing vertex and the "over arc" edges towards the vertex. This choice of orientation will prove useful in what follows.

In particular, it ensures that there is an Euler circuit. Indeed, each vertex of $G_{\mathcal{D}}$ has in-degree two and out-degree two. Since $\mathcal{D}$ is the diagram of a knot, $G_{\mathcal{D}}$ is a connected graph and it follows that $G_{\mathcal{D}}$ has a directed Euler circuit, ie, a closed path that runs through each edge exactly once, and passes through each vertex twice. (For example, see West [15, Theorem 1.4.24].) Fix an Euler circuit $E$ in $G_{\mathcal{D}}$ and a starting edge $e_{1}$. Following $E$, the next edge will be $e_{2}$ and so on. We then write

$$
E=\left(e_{1}, \ldots, e_{2 n}\right)
$$

and subpaths of the Euler circuit will be denoted as subsequences of consecutive elements from the sequence above. 
Let $Y^{\prime}$ be a pseudo coloring as in Lemma 3.1. As we remarked above, $Y^{\prime}$ is not a trivial coloring and therefore has a largest color $h$ and a smallest color $l$ with $0<l<h$. We can carry the coloring of $\mathcal{D}$ over to a coloring of the edges of the digraph $G_{\mathcal{D}}$. Call an edge with color $h$ an $h-e d g e$. Recall that there is a unique crossing, which we will call the +1 -crossing, where $2 x-y-z=1$. Similarly, the -1 -crossing will refer to the unique crossing where $2 x-y-z=-1$. Every other crossing is a $0-$ crossing, ie, $2 x-y-z=0$. We will also call refer to the corresponding vertices of $G_{\mathcal{D}}$ as $+1-$, 0 - or -1 -crossings.

We will now investigate subpaths of the Euler circuit $E$ made up exclusively of $h$-edges.

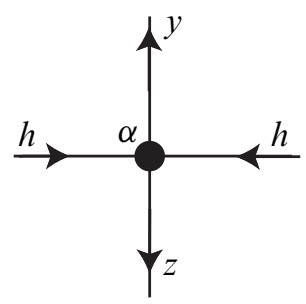

Figure 2: Possible colors at an $\alpha$-crossing ( $\alpha=-1,0$, or 1) where an $h$-edge terminates. Set $y=h-\epsilon$. Since $\alpha=2 h-y-z$, then $z=h+\epsilon-\alpha$.

Let us pick an $h$-edge and follow $E$ starting with this $h$-edge. The possible colors of edges at the vertex where the $h$-edge ends are as illustrated in Figure 2. We may assign one of the outgoing edges the color $h-\epsilon$ (with $\epsilon \geq 0$ ). The other will have color $h+\epsilon-\alpha$. There are three possibilities in order for the $2 x-y-z=\alpha$ condition to hold $(\alpha=0, \pm 1)$ :

- -1-crossing: over arc of color $h$, one of the under arcs of color $h-\epsilon$, and the other under arc of color $h+\epsilon+1>h$, which is impossible.

- 0-crossing: over arc of color $h$, one of the under arcs of color $h-\epsilon$, the other under arc of color $h+\epsilon$, which works provided $\epsilon=0$.

- +1-crossing: over arc of color $h$, one of the under arcs of color $h-\epsilon$, the other under arc of color $h+\epsilon-1$, which works for $\epsilon=0$ or $\epsilon=1$. These amount to the same situation: over $\operatorname{arc} h$; one of the under $\operatorname{arcs} h$; and the other under arc $h-1$.

We thus see that an $h$-edge points to the +1 -crossing or to one of the 0 -crossings, but never to the -1 -crossing. Moreover, if it points to a 0 -crossing then the other edges incident to this vertex will each receive the color $h$ (we'll call such a $0-$ crossing and the corresponding vertex in $G_{\mathcal{D}}$ an $h$-vertex); if it points to the +1 -crossing then 
one of the outgoing edges receives color $h-1$ whereas the remaining edges receive color $h$. In this way, following the Euler circuit $E$ starting from an $h$-edge, one visits $h$-edges incident to $h$-vertices until one reaches an edge incident to the $+1-$ crossing. Here there are two possibilities. Either the next edge is the $h-1$ edge and the visiting of consecutive $h$-edges ends here or the next edge is an $h$-edge and the visiting of consecutive $h$ edges continues.

We remark that any subpath of the Euler circuit which visits only $h$-edges (we'll call it an $h$-path for short) and visits the +1 -crossing twice, must end at this crossing. As a matter of fact, its next edge would be the $h-1$ edge incident to the $+1-$ crossing. Consider then the longest such $h$-path, call it $H$. By shifting the labels of the edges if necessary we have

$$
H=\left(e_{1}, \ldots, e_{n_{H}}\right) \subseteq\left(e_{1}, \ldots, e_{n_{H}}, e_{n_{H}+1}, \ldots, e_{2 n}\right)=E .
$$

Note that $e_{n_{H}}$ is necessarily directed towards the +1 -crossing.

We now argue that each $h$-edge of $E$ is contained in $H$.

Assume to the contrary that $e_{n_{H}+l}$ is an $h$-edge. Then resuming the Euler circuit $E$ as of this edge, either all remaining edges are $h$-edges - which would imply that $e_{2 n}$ is an $h$-edge which would contradict $H$ being the longest $h$-path - or there are edges other than $h$-edges between $e_{n_{H}+l}$ and $e_{2 n}$. But we have already noticed that the progression from an $h$-edge to an edge with a less color goes through the unique +1 -crossing vertex to its $h-1$ edge. However, this step has already been taken; it is $\left(e_{n_{H}}, e_{n_{H}+1}\right)$. Since in an Euler circuit each edge is visited exactly once, then we conclude that there are no $h$-edges past $e_{n_{H}}$.

Let us now observe that $e_{1}$ is the unique $h$-edge at its initial vertex $v_{1}$. Indeed, the edges corresponding to the over arc cannot receive color $h$, for $H$ is the longest $h$-path, and it is straightforward to check that the other edge originating at $v_{1}$ is also not an $h$-edge. Consequently, the vertices with outgoing $h$-edges are $v_{1}, h$-vertices, and the $+1-$ crossing.

Let us call $\mathcal{H}$ the portion of the knot diagram $\mathcal{D}$ which gives rise to the $h$-path $H$ of $E$. That is, $\mathcal{H}$ is the set of $h$-arcs (ie, arcs of color $h$ ) of $\mathcal{D}$ (see Figure 3). We will now argue that $\mathcal{H}$ factors out of $\mathcal{D}$ thereby showing that the knot under consideration is not prime. Let $a_{1}$ be the arc of $\mathcal{H}$ which gives rise to $e_{1} \in H$ and choose an orientation of the knot so that starting at $a_{1}$ one progresses into $\mathcal{H}$. The previous paragraph allows us to conclude that if we follow this orientation of $\mathcal{D}$ starting on an $h$-arc, then we remain on $h$-arcs until we reach either the +1 -crossing or the crossing corresponding to $v_{1}$. 


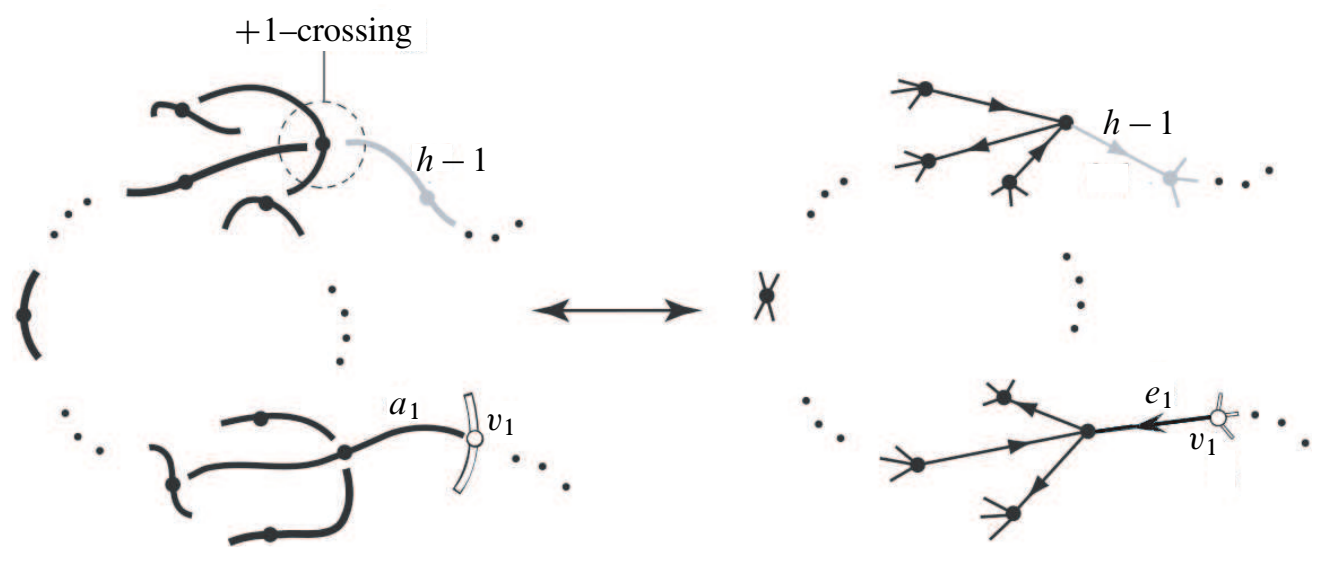

Figure 3: The subpath $H$ of the Euler circuit (at right) and associated component $\mathcal{H}$ of $\mathcal{D}$ (at left). All the black edges and arcs are $h$-edges and $h-\operatorname{arcs}$.

Starting then at $a_{1}$ and following the orientation of the knot we eventually come back to $a_{1}$ so at some point we must have left $\mathcal{H}$. The only way of leaving $\mathcal{H}$ is through the +1 -crossing or the $v_{1}$ crossing. As we have chosen an orientation for $a_{1}$ directed away from the $v_{1}$ crossing, then, reasoning along the same lines as we did for proving there were no $h$-edges outside $H$, we only leave $\mathcal{H}$ once, at the +1 -crossing,

If this portion of the diagram (starting with $a_{1}$ and continuing until we leave $\mathcal{H}$ ) did not go over all the $h$-arcs of $\mathcal{H}$ then starting at any one of the $h$-arcs left out we eventually come back to $a_{1}$. Only this time we never left $\mathcal{H}$ for the only crossings available are those corresponding to $h$-vertices and possibly the over arcs of the $+1-$ crossing. This implies the arc preceding $a_{1}$ is also an $h$-arc which in turn implies that the corresponding vertex $v_{1}$ in $G_{\mathcal{D}}$ has two $h$-edges stemming from it; but this possibility was ruled out above. The contradiction shows that there are no $h$-arcs omitted when we start at $a_{1}$ and follow the orientation until we leave $\mathcal{H}$. In other words, $\mathcal{H}$ corresponds to a summand of the knot under study. Moreover, by extending $\mathcal{H}$ just beyond the $+1-$ crossing if necessary, it is a summand that includes at least one crossing. As $\mathcal{D}$ was a reduced diagram, if follows that $\mathcal{H}$ constitutes a nontrivial summand. Repeating the reasoning above for the least color $l$ we conclude that there is a second nontrivial summand distinct from the preceding one. We have thus proven the following result.

Proposition 3.2 A knot with a diagram endowed with a $Y^{\prime}$ pseudo coloring is not a prime knot.

Finally, we prove our main result on the coloring matrix. 
Proposition 3.3 Let $k$ be an alternating knot with a prime determinant $p$ and $\mathcal{D} \in\left[k_{r}\right]$. Then, every column of the coloring matrix $L$ includes entries that are not zero modulo $p$.

Proof Suppose, to the contrary, that some column of $L$ is zero modulo $p$. Then, by Lemma 3.1 there is a pseudo coloring $Y^{\prime}$ and by Proposition 3.2 this implies $k$ is composite.

However, an alternating knot of prime determinant is prime. To see this, let $k_{1} \# k_{2}$ denote the connected sum of knots $k_{1}$ and $k_{2}$, and let $\Delta_{k}(t)$ be the Alexander polynomial of the knot $k$. Now, $\operatorname{det} k=\left|\Delta_{k}(-1)\right|$ and $\Delta_{k_{1} \# k_{2}}(t)=\Delta_{k_{1}}(t) \Delta_{k_{2}}(t)$ (eg, see Murasugi [11, Chapter 6]). From this it follows that $\operatorname{det}\left(k_{1} \# k_{2}\right)=\operatorname{det} k_{1} \cdot \operatorname{det} k_{2}$. To complete the argument, note that the determinant of a nontrivial alternating knot is not one. For example, the determinant is bounded below by the crossing number (see Burde and Zieschang [3, Proposition 13.30]).

\section{The proof of the Conjecture}

In this section, we prove Conjecture 1 . Let $k$ be an alternating knot of prime determinant and $\mathcal{D} \in\left[k_{r}\right]$. We will say a coloring of $\mathcal{D}$ is heterogeneous if it assigns different colors to different arcs. Thus, we can prove the conjecture by showing that every nontrivial coloring of $\mathcal{D}$ is heterogeneous. There are two steps in the argument. First we show that if one coloring of $\mathcal{D}$ is heterogeneous, then they all are. We conclude the argument by showing that there is such a heterogeneous coloring.

For the first part, we introduce the idea of a fundamental coloring. We say $\mathcal{D}$ has one fundamental coloring if given any two distinct nontrivial colorings $X_{1}$ and $X_{2}$ there are integers $a, b$ such that $X_{2} \equiv a T+b X_{1} \bmod \operatorname{det} k$ where $T$ is the trivial coloring, a vector of all 1 's. There are two immediate consequences.

Lemma 4.1 Let $k$ have prime determinant and suppose $\mathcal{D} \in[k]$ has one fundamental coloring. If a nontrivial coloring assigns different colors to two particular $\operatorname{arcs}$ of $\mathcal{D}$, then every nontrivial coloring will do so. Thus, if $\mathcal{D}$ has a heterogeneous coloring, then every nontrivial coloring of $\mathcal{D}$ is heterogeneous.

So, in order to complete the first part of the argument, it will be enough to show that a diagram of a knot of prime determinant has one fundamental coloring.

Proposition 4.2 Let $k$ be a knot with prime determinant and $\mathcal{D} \in\left[k_{r}\right]$. Then $\mathcal{D}$ has one fundamental coloring. 
Proof Let $k$ have determinant $p$ and $\mathcal{D}$ have $n$ crossings. We will show that the colorings of $\mathcal{D}$ are determined by the colors of two specific arcs in $\mathcal{D}$.

Since $\operatorname{det} k=p$, the determinant of the minor crossing matrix is $\operatorname{det} C= \pm p$. With elementary row operations, and the Euclidean algorithm for finding the gcd of two integers, we can put $C$ in triangular form with integers on the diagonal. Its determinant is then the product of the diagonal entries; all must be \pm 1 except for one, which is $\pm p$. It follows that, $\bmod p$, the rank of $C$ is $n-2$; we'll write $\operatorname{dimim} C=n-2$. Then the nullity, or dimension of the null space, is one; $\operatorname{dim} \operatorname{ker} C=1$.

This means that the dimension of the mod $p$ null space of the crossing matrix $C^{\prime}$ is two. Indeed, $\operatorname{dimim} C^{\prime} \geq \operatorname{dimim} C=n-2$, so $\operatorname{dim} \operatorname{ker} C^{\prime} \leq 2$. On the other hand, the trivial coloring assigning 1 to every arc is not in $\operatorname{ker} C$ but is in $\operatorname{ker} C^{\prime} \bmod p$, so $1=\operatorname{dim} \operatorname{ker} C<\operatorname{dim} \operatorname{ker} C^{\prime} \leq 2$. Therefore, the null space of $C^{\prime} \bmod p$ has dimension two.

We can now demonstrate that there is one fundamental coloring. As the nullity of $C^{\prime}$ is two, every coloring is determined by the coloring of two specific arcs of $\mathcal{D}$. Consider two nontrivial colorings of $\mathcal{D}, X_{1}$ induced by coloring the two $\operatorname{arcs} x_{1}$ and $y_{1}$ and $X_{2}$ induced by the colors $x_{2}$ and $y_{2}$. We can show that there is one fundamental coloring by finding $a$ and $b$ so that $X_{2} \equiv a T+b X_{1}$. Let

$$
a \equiv \frac{y_{1} x_{2}-x_{1} y_{2}}{y_{1}-x_{1}} \quad \text { and } \quad b \equiv \frac{y_{2}-x_{2}}{y_{1}-x_{1}} .
$$

(Since $X_{1}$ is nontrivial, $y_{1} \not \equiv x_{1}$ and $y_{1}-x_{1}$ has an inverse mod $p$.) Then

$$
x_{2} \equiv a \cdot 1+b \cdot x_{1} \quad \text { and } \quad y_{2} \equiv a \cdot 1+b \cdot y_{1},
$$

as required.

Combining Lemma 4.1 and Proposition 4.2 we have completed the first part of our argument:

Corollary 4.3 Let $k$ be a knot with prime determinant and $\mathcal{D} \in\left[k_{r}\right]$. If $\mathcal{D}$ has one heterogeneous coloring, then any nontrivial coloring is heterogeneous.

Remark The corollary applies not only to alternating knots, but to any knot of prime determinant.

It remains to show that there is a heterogeneous coloring.

Proposition 4.4 Let $k$ be an alternating knot with prime determinant. If $\mathcal{D} \in\left[k_{r}\right]$, then $\mathcal{D}$ admits a heterogeneous coloring. 
Proof Let $k$ be an $n$-crossing alternating knot with prime determinant $p$. Construct an oriented labeling for $\mathcal{D}$ and the crossing matrix $C^{\prime}$. Then, by Lemma 2.2, $C^{\prime \top}$ is a crossing matrix for $\mathcal{D}^{m}$ and the minor crossing matrix for $\mathcal{D}^{m}$ is likewise $C^{\top}$. (Note that $\mathcal{D}^{m}$ is also a reduced diagram of an alternating knot of determinant $p$.) Let $L^{m}$ denote the coloring matrix of $\mathcal{D}^{m}$; elementary linear algebra shows $L^{m}=L^{\top}$. Now, Proposition 3.3 demonstrates that all the columns of $L^{\top}$ have nontrivial entries mod $p$. So, for example, in the first column of $L^{\top}$ there is a nonzero entry: $\left(L^{\top}\right)_{i 1} \not \equiv 0$. That is, using Lemma 2.1, the first column extends to a coloring that distinguishes $a_{i}$ and $a_{n}$ in $\mathcal{D}^{m}$.

Then, by Lemma 4.1 and Proposition 4.2, all nontrivial colorings distinguish $a_{i}$ and $a_{n}$ in $\mathcal{D}^{m}$. Hence $\left(L^{\top}\right)_{i j} \not \equiv 0$ for $j=1, \ldots, n-1$. That is, the $i$-th row of $L^{\top}$, or equivalently, the $i$-th column of $L$ consists only of nonzero entries modulo $p$. Therefore, the $i$-th column of $L$ extends to a coloring differentiating $a_{n}$ from all other $\operatorname{arcs}$ in $\mathcal{D}$ :

$$
X^{\prime}=\left(\begin{array}{c}
(L)_{i 1} \\
\vdots \\
(L)_{i(n-1)} \\
0
\end{array}\right)
$$

Now, since an oriented labeling can start at any arc, the arc labeled with $a_{n}$ is arbitrary. Hence, by repeating the above argument while shifting the oriented labeling, we can exhibit a coloring differentiating any arc from all other arcs. But, by Proposition 4.2, $k$ has one fundamental coloring, so, by Lemma 4.1, every nontrivial coloring differentiates the same arcs. Therefore the coloring given in (5) must be heterogeneous.

Together, Corollary 4.3 and Proposition 4.4 prove the conjecture.

Acknowledgement We thank the referee for many helpful comments that greatly improved the exposition.

\section{References}

[1] C C Adams, The knot book. An elementary introduction to the mathematical theory of knots, Amer. Math. Soc. (2004) MR2079925

[2] M M Asaeda, J H Przytycki, A S Sikora, Kauffman-Harary conjecture holds for Montesinos knots, J. Knot Theory Ramifications 13 (2004) 467-477 MR2069036

[3] G Burde, H Zieschang, Knots, second edition, de Gruyter Studies in Math. 5, de Gruyter, Berlin (2003) MR1959408 
[4] N E Dowdall, T W Mattman, K Meek, P R Solis, On the Harary-Kauffman Conjecture and Turk's head knots, to appear in Kobe J. Math. arXiv:0811.0044

[5] F Harary, L H Kauffman, Knots and graphs. I. Arc graphs and colorings, Adv. in Appl. Math. 22 (1999) 312-337 MR1675756

[6] L H Kauffman, State models and the Jones polynomial, Topology 26 (1987) 395-407 MR899057

[7] L H Kauffman, S Lambropoulou, On the classification of rational tangles, Adv. in Appl. Math. 33 (2004) 199-237 MR2074397

[8] C Livingston, Knot theory, Carus Math. Monogr. 24, Math. Ass. Amer., Washington, DC (1993) MR1253070

[9] M K Mo, Knot colorings and the Alexander polynomial, Lecture notes (2007)

[10] K Murasugi, Jones polynomials and classical conjectures in knot theory, Topology 26 (1987) 187-194 MR895570

[11] K Murasugi, Knot theory and its applications, Birkhäuser, Boston (1996) MR1391727 Translated from the 1993 Japanese original by B Kurpita

[12] L Person, M Dunne, J DeNinno, B Guntel, L Smith, Colorings of rational, alternating knots and links, Preprint (2002)

[13] J H Przytycki, 3-coloring and other elementary invariants of knots, from: "Knot theory (Warsaw, 1995)", (V F R Jones, J Kania-Bartoszyńska, J H Przytycki, P Traczyk, V G Turaev, editors), Banach Center Publ. 42, Polish Acad. Sci., Warsaw (1998) 275295 MR1634462

[14] M B Thistlethwaite, A spanning tree expansion of the Jones polynomial, Topology 26 (1987) 297-309 MR899051

[15] D B West, Introduction to graph theory, Prentice Hall, Upper Saddle River, NJ (1996) MR1367739

Department of Mathematics and Statistics, California State University, Chico

Chico, CA 95929-0525, USA

Department of Mathematics, University of California

Berkeley, CA, USA

TMattman@CSUChico.edu, pablo@math.berkeley.edu

Received: 14 February 2008 Revised: 17 July 2009 